\title{
Prevalencia de tromboembolia pulmonar en pacientes intervenidos de cirugía abdominopélvica con dificultad respiratoria
}

\author{
Prevalence of pulmonary thromboembolism in patients undergoing abdominopelvic \\ surgery with respiratory distress
}

\author{
Carlos A. López-Bernal', Eduardo Vázquez-Cruz², Aurelio López-Colombo ${ }^{1}$, Álvaro J. Montiel-Jarquín ${ }^{1 *}$, \\ Felipe Cruz-Vega ${ }^{3}$ y Jorge Loria-Castellanos ${ }^{3}$ \\ ${ }^{1}$ Unidad Médica de Alta Especialidad Hospital de Especialidades de Puebla, Instituto Mexicano del Seguro Social (IMSS), Puebla; ${ }^{2}$ Unidad de \\ Medicina Familiar No. 6 en Puebla, IMSS, Puebla; ${ }^{3}$ División de Proyectos Especiales en Salud del IMSS, Ciudad de México. México
}

\begin{abstract}
Resumen
Objetivo: Determinar la prevalencia de la tromboembolia pulmonar (TEP) e identificar sus factores de riesgo en los pacientes intervenidos en el servicio de cirugía general de manera programada de la Unidad Médica de Alta Especialidad Hospital de Especialidades de Puebla, del Instituto Mexicano del Seguro Social. Método: Estudio descriptivo, retrospectivo y transversal de 52 expedientes de pacientes con TEP y angiotomografía pulmonar cuya indicación fue la dificultad respiratoria, de 2,612 pacientes operados de noviembre de 2015 a marzo de 2018. El muestreo fue no probabilístico. La estadística fue descriptiva, con medidas de tendencia central y de dispersión. Se utilizó prueba de Fisher para riesgo y Rho de Spearman. Resultados: Hubo asociación entre el desarrollo de TEP y el tiempo transquirúrgico $(p=0.018)$, y con el dímero $D(p=0.014)$; hubo correlación entre la TEP y con la escala de Wells $(p=0.001)$ y con la transfusión de hemoderivados $(p=0.044)$. La edad, el sexo, el índice de masa corporal, el diagnóstico preoperatorio, la tromboprofilaxis, la transfusión de hemoderivados y el abordaje quirúrgico no fueron factores de riesgo. Conclusión: La prevalencia de TEP posquirúrgica en pacientes que cursan con dificultad respiratoria después del procedimiento quirúrgico es del $5.7 \%$ y la mortalidad es del $33.3 \%$.
\end{abstract}

Palabras Clave: Cirugía general. Prevalencia. Tromboembolia pulmonar.

\begin{abstract}
Objective: To determine the prevalence of pulmonary thromboembolism (PET) and identify it's risk factors in patients operated on a scheduled basis, in the general surgery service from the High Specialty Medical Unit Hospital de Especialidades de Puebla, of the Mexican Social Security Institute. Method: Descriptive, retrospective and cross sectional study of 52 patients' records with PET and pulmonary angiotomography whose indication was respiratory distress; from 2,612 operated patients from November 2015 to March 2018, the sampling was not probabilistic. The statistics was descriptive, with measures of central tendency and dispersion. Fisher test, and Rho Spearman were used. Results: There were association between the development of PET and trans-surgical time $(p=0.018)$, with the D Dimer $(p=0.014)$, there was correlation between PET and Wells scale $(p=0.001)$ and blood transfusion $(p=0.044)$. Age, gender, body mass index, preoperative diagnosis, thrombo-
\end{abstract}

Correspondencia:

*Álvaro J. Montiel-Jarquín

Calle 2 norte 2004, Centro

Fecha de recepción: 06-11-2018

Cir Cir. 2020;88(1):20-26

C.P. 72000 , Puebla, México

Fecha de aceptación: 08-02-2019

tents available at PubMed

E-mail: dralmoja@hotmail.com

DOI: $10.24875 / C I R U .19000874$

www.cirugiaycirujanos.com

0009-7411/@ 2019 Academia Mexicana de Cirugía. Publicado por Permanyer. Éste es un artículo open access bajo la licencia CC BY-NC-ND (http://creativecommons.org/licenses/by-nc-nd/4.0/). 
prophylaxis, blood transfusion and surgical approach were not risk factors. Conclusion: The prevalence of postoperative PET in patients with respiratory distress after surgical procedure is $5.7 \%$ and the mortality is $33.3 \%$.

Key Words: General surgery. Prevalence. Pulmonary thromboembolism.

\section{Introducción}

La tromboembolia pulmonar (TEP) es una manifestación clínica que consiste en la obstrucción mecánica a la circulación pulmonar por un trombo o émbolo en un ambiente de hipercoagulabilidad y daño endotelial. Es la complicación más temida de la enfermedad tromboembólica venosa'.

La TEP se encuentra asociada a factores de riesgo congénitos, como disfibrinogenemia, déficit de antitrombina, déficit de proteína $\mathrm{C}$ o déficit de proteína $\mathrm{S}$, y adquiridos, como cirugía mayor, edad avanzada, inmovilización prolongada, cáncer o trombosis previa, que condicionan daño endotelial y que explican su fisiopatología $a^{2-4}$.

La importancia de reconocer estos factores de riesgo tiene como elemento principal el prevenirlos de ser posible y desarrollar estrategias para disminuir la incidencia de la TEP ${ }^{5-7}$. El estado de hipercoagulabilidad y el daño endotelial se ha demostrado que están presentes hasta 28 días después de la cirugía y aún pueden condicionar TEP8.

La prevalencia de la TEP en pacientes operados es incierta, pero se estima que es del $5-25 \%$ en pacientes asociados a eventos quirúrgicos, cifra equiparable a la incidencia de la misma enfermedad ${ }^{9}$. La cirugía abdominal engloba procedimientos quirúrgicos que son factor de riesgo para el desarrollo de TEP; se puede asociar hasta en un $6 \%$ como causa directa de la misma ${ }^{10}$.

Para el diagnóstico de TEP, la angiotomografía pulmonar se ha convertido en el estándar de oro. En pacientes con una probabilidad clínica baja o intermedia de TEP, valorada mediante la regla de Wells, un resultado negativo tiene un alto valor predictivo negativo (VPN) de hasta el $96 \%$, mientras que solo el $60 \%$ tiene una probabilidad alta. Por el contrario, el valor predictivo positivo (VPP) de una angiotomografía pulmonar positiva es alto (92-96\%) en pacientes con probabilidad clínica intermedia o alta, pero mucho menor $(58 \%)$ en pacientes con baja probabilidad previa a la prueba de TEP ${ }^{11}$.

El tratamiento de elección en los pacientes con TEP sigue siendo la anticoagulación, así como el soporte vital de la mecánica ventilatoria y de la hemodinamia. Se recomienda la anticoagulación para prevenir tanto la muerte precoz como la TEP sintomática recurrente o mortal. La duración estándar de la anticoagulación debe cubrir al menos 3 meses $^{12}$.

Conocer la prevalencia de la TEP en los pacientes operados en el servicio de cirugía general es de utilidad para mejorar y proponer medidas profilácticas adaptadas a las necesidades del hospital, así como a la población promedio atendida en dicho servicio, además de optimizar el uso de recursos y ofrecer mejores resultados posquirúrgicos a los pacientes, disminuyendo la morbimortalidad de los eventos trombóticos.

El objetivo del presente trabajo fue determinar la prevalencia de la TEP e identificar sus factores de riesgo en el servicio de cirugía general de la Unidad Médica de Alta Especialidad (UMAE) del Instituto Mexicano del Seguro Social (IMSS) de Puebla, en el período comprendido entre noviembre de 2015 y marzo de 2018. Todos los pacientes contaron con angiotomografía pulmonar, cuya indicación fue dificultad respiratoria después de procedimientos quirúrgicos programados, y formaron parte del protocolo diagnóstico de TEP.

Este estudio cumplió con los lineamientos éticos siguientes: fue aprobado por los comités locales de investigación y ética en investigación en salud de la unidad médica participante, fue diseñado de acuerdo a la Ley general de salud en materia de investigación, Norma técnica número 313, Reglamento federal título 15, además de la Declaración de Helsinki, así como a la Norma Oficial Mexicana NOM-012-SSA3-2012. No se procedió al llenado de consentimiento informado debido a que los datos fueron recolectados del expediente clínico.

\section{Método}

Se realizó un estudio descriptivo, retrospectivo y transversal en el que se analizaron expedientes clínicos solo de pacientes que contaban con angiotomografía pulmonar y que tras la cirugía abdominal de forma electiva presentaron dificultad respiratoria en la UMAE, Hospital de Especialidades de Puebla, Centro Médico Nacional General de División Manuel Ávila Camacho, del IMSS, de noviembre de 2015 a marzo de 2018. 
El marco muestral fue no probabilístico y el tamaño de la muestra fue conveniente al investigador, dado por el total de pacientes que cumplieron con los criterios de inclusión en el período de estudio y para eliminar sesgo a la toma de muestra. La población elegible fue de 2,612 pacientes operados de manera electiva durante el período de estudio. De este total de pacientes, se seleccionaron 52 operados de cirugía abdominal que contaban con angiotomografía pulmonar y presentaron dificultad respiratoria posquirúrgica como indicación de la misma. Se incluyeron pacientes de ambos sexos, derechohabientes del IMSS, con TEP diagnosticada por angiotomografía pulmonar, con expediente clínico completo. Se excluyeron pacientes intervenidos en otra unidad hospitalaria, con diagnóstico previo de TEP. Por el tipo de estudio, no hubo criterios de eliminación, y de igual manera no se generó hipótesis.

Para el estudio se emplearon las siguientes variables: diagnóstico prequirúrgico, sexo, edad, índice de masa corporal, tromboprofilaxis mecánica, tromboprofilaxis medicamentosa, puntaje de la escala de Wells, angiotomografía pulmonar, dímero $D$, escala American Scociety Anestesiology (ASA), abordaje quirúrgico, tiempo de cirugía en minutos, sangrado transquirúrgico en mililitros, comorbilidad asociada a la patología quirúrgica y diagnósticos diferenciales.

\section{Resultados}

Se revisaron 2,612 pacientes, de los cuales 52 cumplieron con los criterios de selección. La edad de los pacientes fue de 50.4 años, con un mínimo de 28 y un máximo de 76 ( \pm 13.5 años). La distribución del sexo fue del $50 \%$ hombres y el $50 \%$ mujeres $(n=26$ cada uno).

Como medida antropométrica, se midió el índice de masa corporal y se encontró una media de 28.18, con un mínimo de 21 y un máximo de 39 ( \pm $4.2974 \mathrm{~kg} / \mathrm{m}^{2}$ ). En cuanto a los diagnósticos prequirúrgicos de los pacientes, el más frecuente fue el cáncer de recto, con 14 (26.9\%) pacientes, seguido del cáncer de colon y el cáncer de páncreas con $7(13.5 \%)$ pacientes cada uno, y en tercer lugar el cáncer de gástrico con 4 (7.7 \%) pacientes; el resto se muestra en la tabla 1.

De las intervenciones realizadas, el abordaje quirúrgico fue abierto en 43 (82.7\%) y laparoscópico en $9(17.3 \%)$ pacientes. Entre las comorbilidades que tenían los pacientes, la diabetes mellitus tipo 2 y la hipertensión arterial sistémica fueron las más
Tabla 1. Diagnósticos de los pacientes incluidos en el estudio

\begin{tabular}{lcc}
\hline Diagnóstico & $\mathbf{n}$ & $\%$ \\
\hline Cáncer de recto & 14 & 26.9 \\
Cáncer de colon & 7 & 13.5 \\
Cáncer de páncreas & 7 & 13.5 \\
Cáncer gástrico & 6 & 11.5 \\
Cáncer de esófago & 4 & 7.7 \\
Perforación intestinal & 9 & 17.3 \\
Colecistitis litiásica & 4 & 7.7 \\
Enfermedad por reflujo gastroesofágico & 5 & 9.6 \\
$N$ & 52 & 100 \\
\hline
\end{tabular}

Tabla 2. Comorbilidad de los pacientes con dificultad respiratoria posquirúrgica

\begin{tabular}{llc}
\hline Enfermedad & $\mathbf{n}$ & $\%$ \\
\hline Diabetes mellitus tipo 2 & 16 & 30.8 \\
Hipertensión arterial & 16 & 30.8 \\
Cardiopatía isquémica & 6 & 11.5 \\
Enfermedad renal crónica & 4 & 7.7 \\
Enfermedad pulmonar obstructiva crónica & 1 & 1.9 \\
Sin comorbilidad & 9 & 17.3 \\
Total & 52 & 100 \\
\hline
\end{tabular}

frecuentes, con $16(30.8 \%)$ cada una; el resto se muestra en la tabla 2.

El riesgo quirúrgico de los pacientes fue calculado con la escala ASA. Se observó ASA I en 2 (3.8\%), ASA II en 46 (88.5\%) y ASA III en 4 (7.7\%) pacientes. El tiempo transquirúrgico de la cirugía fue una media de 164.9 minutos, con un mínimo de 55 y un máximo de 320 ( \pm 69.6 minutos). El número de pacientes que necesitaron hemoderivados fue 19 (36.5\%), mientras que $33(63.5 \%)$ no los ameritaron.

El sangrado transquirúrgico medio observado fue de $541.4 \mathrm{ml}$, con un mínimo de 5 y un máximo de 1,900 $( \pm 480.7 \mathrm{ml}$ ). La media del dímero $\mathrm{D}$ después de la dificultad respiratoria aguda fue de $1697.96 \mu \mathrm{g} / \mathrm{l}$, con un mínimo de 670 y un máximo de 3,452 ( \pm 812.1 $\mu \mathrm{g} / \mathrm{l})$. En cuanto a la escala de Wells como sospecha de TEP, en 19 pacientes fue 2 (36.5\%) puntos, en $28(53.8 \%)$ pacientes fue 3 puntos y en $5(9.6 \%)$ pacientes fue 4 puntos.

Las medidas profilácticas observadas fueron la tromboprofilaxis mecánica con vendas de 
Tabla 3. Etiología de la dificultad respiratoria posquirúrgica

\begin{tabular}{lcc}
\hline Diagnóstico & $\mathbf{n}$ & $\%$ \\
\hline Atelectasia pulmonar & 23 & 44.2 \\
Edema agudo de pulmón & 6 & 11.5 \\
Mediastinitis & 5 & 9.6 \\
Sepsis abdominal & 15 & 28.8 \\
Tromboembolia pulmonar & 3 & 5.7 \\
N & 52 & 100 \\
\hline
\end{tabular}

Tabla 4. Asociación entre sexo, escala de Wells, transfusión de paquetes globulares y abordaje quirúrgico con el desarrollo de tromboembolia pulmonar

\begin{tabular}{|c|c|c|c|c|c|}
\hline \multirow[t]{2}{*}{ Variable } & \multicolumn{2}{|c|}{ Tromboembolia } & \multirow[t]{2}{*}{ Total } & \multirow[t]{2}{*}{ Fisher } & \multirow[t]{2}{*}{$p$} \\
\hline & Sí & No & & & \\
\hline \multicolumn{6}{|l|}{ Sexo } \\
\hline Masculino & 1 & 25 & 26 & & \\
\hline Femenino & 2 & 24 & 26 & & 0.5 \\
\hline Total & 3 & 49 & 52 & & \\
\hline \multicolumn{6}{|l|}{ Escala de Wells } \\
\hline 2 puntos & 0 & 19 & 19 & & \\
\hline 3 puntos & 0 & 28 & 28 & & 0.001 \\
\hline 4 puntos & 3 & 2 & 5 & & \\
\hline Total & 3 & 49 & 52 & & \\
\hline \multicolumn{6}{|l|}{$\begin{array}{l}\text { Transfusión de } \\
\text { hemoderivados }\end{array}$} \\
\hline Sí & 3 & 16 & 19 & & \\
\hline No & 0 & 33 & 33 & & 0.044 \\
\hline Total & 3 & 49 & 52 & & \\
\hline \multicolumn{6}{|c|}{ Abordaje quirúrgico } \\
\hline Abierto & 3 & 40 & 43 & & \\
\hline Laparoscópico & 0 & 9 & 9 & & 0.558 \\
\hline Total & 3 & 49 & 52 & & \\
\hline
\end{tabular}

Tabla 5. Correlación entre la edad, el índice de masa corporal, el dímero $\mathrm{D}$, el tiempo de cirugía y el sangrado con el desarrollo de tromboembolia pulmonar

\begin{tabular}{lcc}
\hline $\begin{array}{l}\text { Variables y desarrollo o no de } \\
\text { tromboembolia pulmonar }\end{array}$ & Rho de Spearman & $\mathbf{p}$ \\
\hline Edad de los pacientes & -0.233 & 0.816 \\
Índice de masa corporal & -0.605 & 0.548 \\
Dímero D & -2.540 & 0.014 \\
Tiempo de cirugía (min) & -2.451 & 0.018 \\
Sangrado transquirúrgico $(\mathrm{ml})$ & -0.248 & 0.077 \\
\hline
\end{tabular}

compresión, utilizada en todos los pacientes; la tromboprofilaxis con medicamentos, en la que se utilizó enoxaparina, fue realizada en $12(23.1 \%)$ pacientes de manera preoperatoria y en $30(57.7 \%)$ pacientes de manera posoperatoria; en 10 (19.2\%) pacientes no se utilizaron medicamentos.

Como causa final para la dificultad respiratoria se encontró atelectasia pulmonar en $23(44.2 \%)$ pacientes y edema agudo de pulmón en 6 (28.8\%) pacientes; el resto se muestra en la tabla 3.

La comparación de las medias del tiempo quirúrgico entre los pacientes que desarrollaron y no desarrollaron TEP fue estadísticamente significativa $(p=0.018$, Spearman). Así mismo, para ver la asociación entre la transfusión de hemoderivados y la aparición de TEP en los pacientes con dificultad respiratoria posquirúrgica se realizó una comparación mediante tablas de contingencia de $2 \times 2$ con prueba exacta de Fisher, en donde se obtuvo una diferencia significativa $(p=0.044)$ entre los pacientes que presentaron TEP y los que no. Con respecto al dímero D, se obtuvo un valor estadísticamente significativo mediante la correlación de Spearman $(p=0.014)$. En cuanto a la escala de Wells como escala de sospecha para el desarrollo de TEP, mediante la prueba exacta de Fisher se obtuvo $p=0.001$ y se consideró estadísticamente significativo ( $p \leq 0.05)$ (Tablas 4 y 5$)$ (Fig. 1).

No se encontraron variables como factor de riesgo para el desarrollo de TEP (Fig. 2).

\section{Discusión}

En el presente estudio se determinó la prevalencia de TEP en pacientes intervenidos en el servicio de cirugía general en el entorno de cirugía abdominal y que posterior al procedimiento presentaron dificultad respiratoria.

La edad media de los pacientes se encontró en la quinta década, que comparado con otros estudios es menor. El estudio de Parry, et $a l .^{13}$ y el de Melloul, et al..$^{14}$ observaron una media de edad de sus pacientes entre 10 y 15 años mayor que en este estudio. El estudio de Jeremitsky, et al. ${ }^{15}$ tuvo una edad similar a la que presentaron los pacientes de nuestra muestra $^{16}$. En dichos estudios, la edad no es tomada como un factor de riesgo, exceptuando el estudio de Stroh, et $a{ }^{16}{ }^{16}$, en el que se observó una mayor prevalencia de TEP en personas mayores. Contrario a lo observado por Stroh, et al. ${ }^{16}$, dentro del presente estudio no se observaron diferencias ni asociaciones de la edad con el desarrollo de TEP.

En cuanto al sexo, la muestra estuvo constituida por la misma cantidad de hombres que de mujeres, diferente a lo observado en el estudio de Parry, et al. ${ }^{13}$, que tuvo una menor cantidad de mujeres que de 


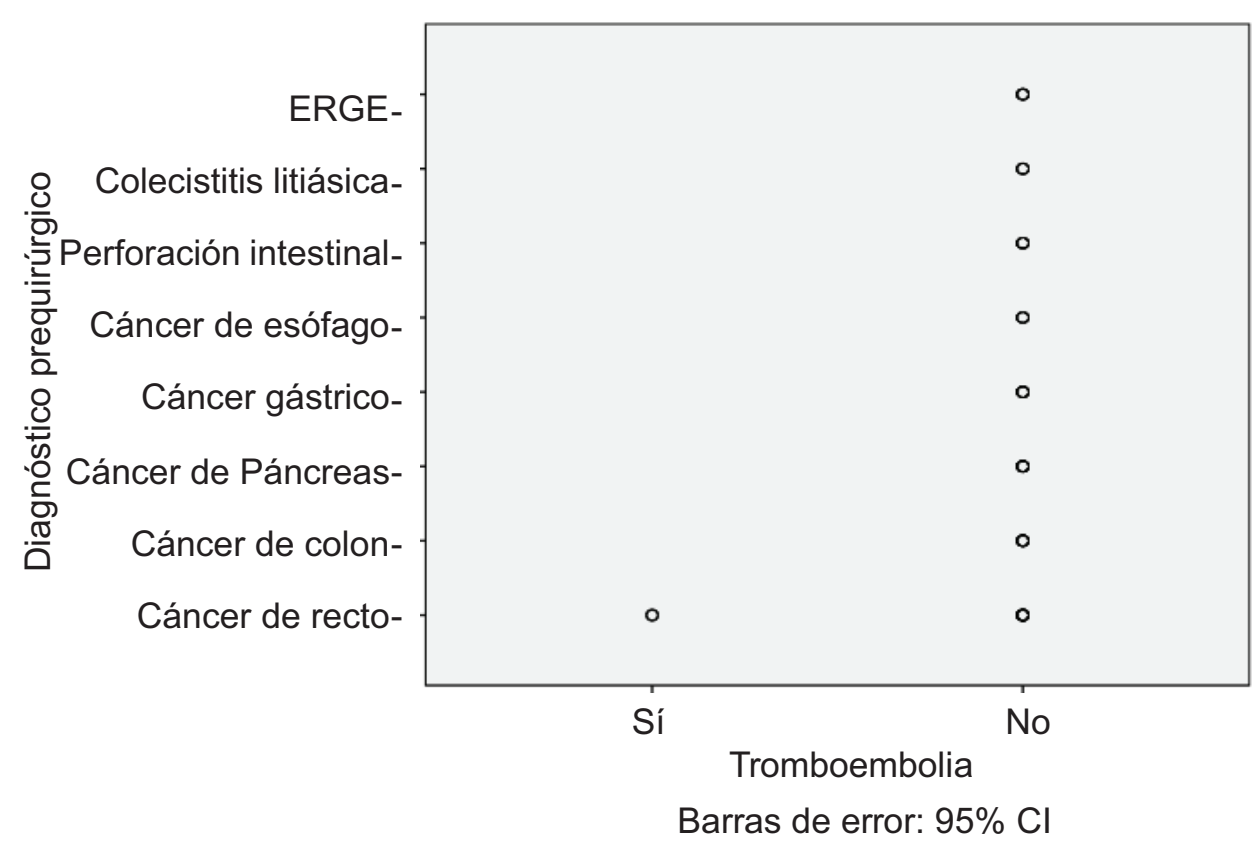

Figura 1. Correlación entre el diagnóstico preoperatorio y el desarrollo de tromboembolia pulmonar.

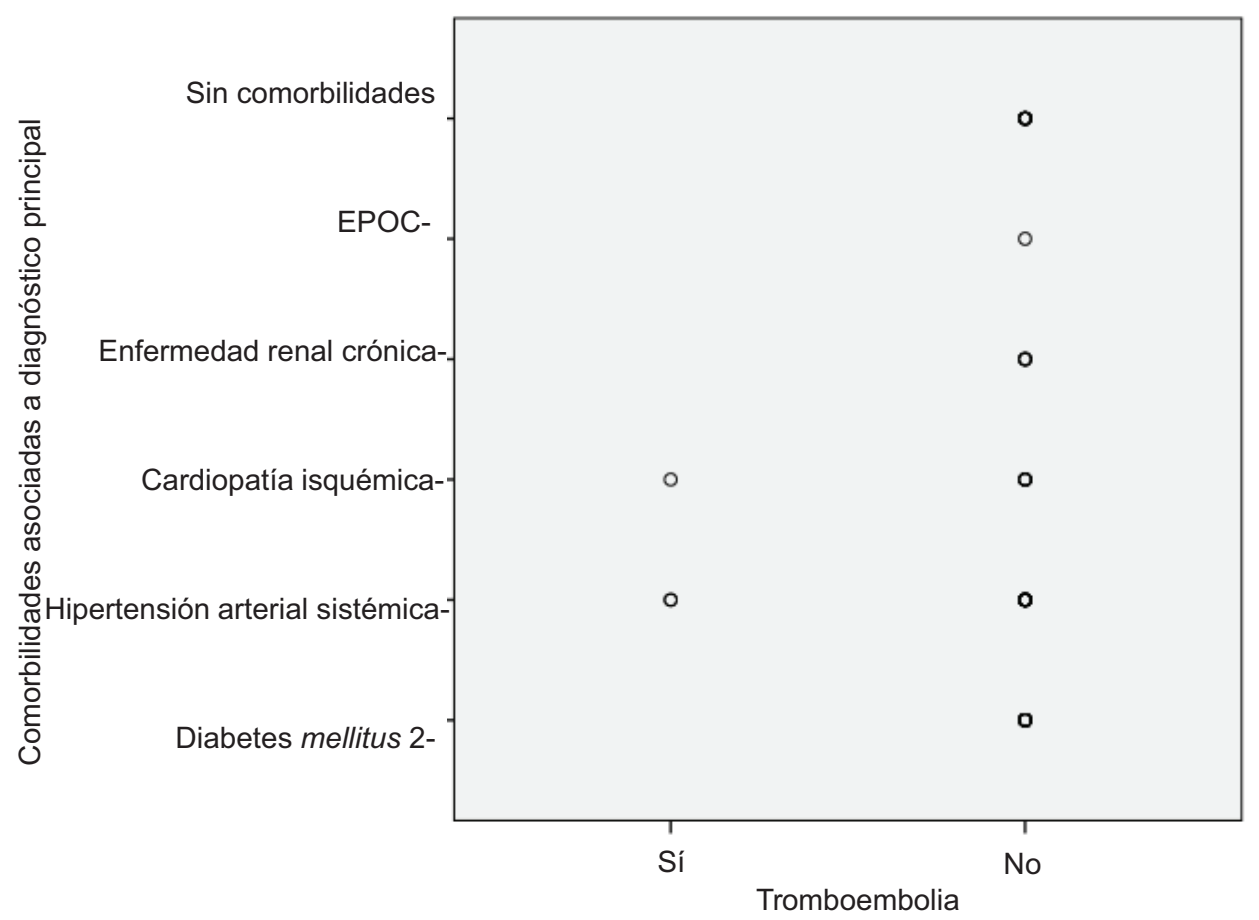

Figura 2. Correlación entre las comorbilidades asociadas al diagnóstico principal y el desarrollo de tromboembolia pulmonar.

hombres. Sin embargo, no se observaron asociaciones con el sexo, similar a lo observado por Melloul, et al..$^{14} \mathrm{y}$ consistente con lo observado en el presente estudio, en el que el sexo no tuvo una asociación con la presencia de TEP. Otro estudio, el de Jeremitsky, et al. ${ }^{15}$, observó que los eventos trombóticos eran más frecuentes en las mujeres que en los hombres.
Entre las cirugías, dentro del estudio, todas fueron electivas y la mayoría de los pacientes tuvieron un procedimiento abierto; esta variable no estuvo asociada con la presencia de TEP. Sin embargo, de manera contraria el estudio de Melloul, et al. ${ }^{14}$, sí se observaron asociaciones entre las cirugías y la presencia de TEP, encontrando que en las resecciones 
mayores en cirugía abierta hubo mayor prevalencia de TEP ${ }^{14}$.

Las dos comorbilidades más frecuentes fueron la hipertensión arterial sistémica y la diabetes mellitus tipo 2, pero estas no se encontraron asociadas con el desarrollo de TEP en los pacientes, de modo similar a los resultados del estudio de Stroh, et al. ${ }^{16}$, quienes también encontraron como comorbilidades frecuentes la hipertensión y la diabetes tipo 2, y de igual manera no tuvieron una asociación estadísticamente significativa. En cuanto a las diferencias, su estudio encontró la apnea del sueño como una comorbilidad, pero esta no fue observada entre los pacientes del presente estudio. Contrario a lo observado, el estudio de Kokudo, et al. ${ }^{17}$ encontró también comorbilidades como enfermedad pulmonar obstructiva crónica, cardiopatía isquémica, tabaquismo y alcoholismo, variables que no se observaron en este estudio.

El riesgo quirúrgico que más se observó en los pacientes fue el ASA II, que no se vio asociado con la presencia de TEP, lo cual es consistente con lo que observaron Kokudo, et al. ${ }^{17}$ en su estudio, en donde el riesgo quirúrgico no estuvo asociado como un factor de riesgo.

El tiempo quirúrgico tuvo una media de 164 minutos, y esta variable se encontró correlacionada con la presencia de TEP. En los pacientes que tuvieron TEP, el tiempo de las cirugías fue estadísticamente mayor que en los pacientes que no tuvieron TEP ( $p=0.10)$. Esto fue similar a lo que encontraron Kokudo, et al..$^{17}$ en su estudio, en donde también se vieron asociados el tiempo quirúrgico y la presencia de TEP. Este resultado es diferente a lo reportado por Parry et al. $^{13}$, quienes no encontraron una asociación entre ambas variables. El sangrado transquirúrgico no fue una variable que se observara correlacionada con la presencia de TEP, lo cual fue similar a lo observado por Parry, et al. ${ }^{13}$.

En cuanto a la escala de Wells, la mayoría de los pacientes presentaron un puntaje de 3 . Al realizar un análisis estadístico, se observó que la media de esta era diferente en los pacientes con TEP que en aquellos que no la presentaron. Entre las medidas profilácticas, todos los pacientes recibieron profilaxis mecánica con medias de mediana compresión, y los medicamentos fueron empleados en la mayoría de los pacientes; esta variable no se asoció con la aparición de TEP.

La prevalencia de TEP en pacientes con dificultad respiratoria posquirúrgica fue del $5.7 \%$, que es más elevada que la encontrada por Wang, et al. ${ }^{18}$ en su estudio, en donde la TEP represento el $0.46 \%$ de los eventos trombóticos posquirúrgicos.

El dímero $D$ como prueba realizada en los pacientes con dificultad respiratoria aguda mostró una sensibilidad del $95 \%$, una especificidad del $5 \%$, un VPN del $94 \%$ y un VPP del $51 \%$. A pesar de ser significativamente estadístico en nuestro estudio, tiene baja especificidad y resulta ser muy sensible. Con respecto a la escala de Wells como escala de probabilidad de TEP, esta ofrece una sensibilidad y una especificidad en nuestro estudio del $50 \%$, un VPN del $94 \%$ y un VPP del $6 \%$, resultando ser una prueba adecuada para el protocolo diagnóstico de los pacientes con sospecha de TEP. En relación a la angiotomografía pulmonar como estudio de referencia, obtuvimos mediante la misma una sensibilidad del $100 \%$ y una especificidad del $50 \%$, un VPP del $6 \%$ y un VPN del $100 \%$; con ello se confirma la prueba como método de referencia para el diagnóstico de TEP.

De igual manera, se compararon las medias de edad, el índice de masa corporal, la cantidad de sangrado, el sexo, el diagnóstico preoperatorio, el riesgo quirúrgico con la escala ASA, el sangrado transquirúrgico, el tipo de abordaje y la comorbilidad, y no fueron diferentes entre los pacientes con dificultad respiratoria posquirúrgica que desarrollaron TEP y los que no la desarrollaron.

Con base en estos resultados se propone evitar tiempos quirúrgicos prolongados y el uso inadecuado de transfusiones sanguíneas, utilizar la escala de Wells para identificar a los pacientes con posibilidades de TEP, y determinar el dímero $D$ a los pacientes con riesgo de TEP. Se recomienda usar medidas profilácticas, como heparina de bajo peso molecular, de manera prequirúrgica siempre que no exista contraindicación alguna, y el uso sistemático de medias de compresión mediana en los miembros inferiores, así como movilizar tempranamente a los pacientes.

\section{Conclusión}

La prevalencia de TEP posquirúrgica en los pacientes que cursan con dificultad respiratoria después del procedimiento quirúrgico es del $5.7 \%$ y la mortalidad es del $33.3 \%$. No se encontraron factores de riesgo para el desarrollo de TEP.

\section{Conflicto de intereses}

Los autores declaran que no hay conflicto de intereses.

\section{Financiamiento}

Este trabajo de investigación no recibió financiamiento por ninguna institución para su realización. 


\section{Responsabilidades éticas}

Protección de personas y animales. Los autores declaran que los procedimientos seguidos se conformaron a las normas éticas del comité de experimentación humana responsable y de acuerdo con la Asociación Médica Mundial y la Declaración de Helsinki.

Confidencialidad de los datos. Los autores declaran que han seguido los protocolos de su centro de trabajo sobre la publicación de datos de pacientes.

Derecho a la privacidad y consentimiento informado. Los autores han obtenido el consentimiento informado de los pacientes y/o sujetos referidos en el artículo. Este documento obra en poder del autor de correspondencia.

\section{Bibliografía}

1. Kozek LS, Fenger EC, Thienpont E, Baraurkas G. European guidelines on perioperative venous thromboembolism prophylaxys. Eur J Anaesthesiol. 2017;34:1-7.

2. Cabrera RA, Nellen HH. Epidemiología de la enfermedad tromboembólica venosa. Gac Med Mex. 2007;143:3-5.

3. Wakefield TW, McLafferty RB, Lohr JM, Caprini JA, Gillespie DL, Passman MA. Call to action to prevent venous thromboembolism. J Vasc Surg. 2009;49:1620-3.

4. Palta S, Saroa R, Palta A. Overview of the coagulation system. Indian J Anaesth. 2014:58:515-23.

5. Imigo FG, Castillo EF, Sánchez AH. Profilaxis de la enfermedad tromboembólica en cirugía. Cuad Cir 2010;24:34-9.

6. Bahl V, Mei HH, Henke KP, Wakefield WT, Campbell AD, Caprini AJ. A validation study of a retrospective venous thromboembolism risk scoring method. Ann Surg. 2010;251:344-50.
7. Marti C, John G, Konstantinides S, Combescure C, Sanchez O, Lankeit $\mathrm{M}$, et al. Systemic thrombolytic therapy for acute pulmonary embolism: a systematic review and meta-analysis. Eur Heart J. 2015; 36:605-14.

8. Ulrych J, Kvasnicka T, Fryba V, Komarc M, Malikova I, Burget F, et al. 28 day post-operative persisted hypercoagulability after surgery for benign diseases: a prospective cohort study. BMC Surg. 2016; 16:1-8.

9. Albores AR, Nava ZA, Guerra SA, Plascencia OT, Rubio JB. Sobre la tromboembolia pulmonar. El residente. 2014;9:124-7.

10. Sakon M, Kakkar AK, Ikedal M, Sekimoto M, Nakamori S, Yano M, et al. Current status of pulmonary in general surgery in Japan. Surg Today. 2004;34:905-10.

11. Horii $\mathrm{Y}$, Yoshimura N, Hori $\mathrm{Y}$, Takaki S, Takano T, Inagawa S, et al. Correlation between the site of pulmonary embolism and the extent of deep vein thrombosis: evaluation by computed tomography pulmonary angiography and computed tomography venography. Jap J Radiol. 2011; 29:171-6.

12. Agnelli G, Buller RH, Cohen A, Curto M, Gallus SA, Johnson M, et al. Oral apixaban for the treatment of acute venous thromboembolism. N Engl J Med. 2013;369:799-808.

13. Parry K, Sadeghi AH, Van Der Horst S, Westerink J, Ruurda JP, Van Hillegersberg R. Intermittent pneumatic compression in combination with low-molecular weight heparin in the prevention of venous thromboembolic events in esophageal cancer surgery. J Surg Oncol. 2017;115: 181-5.

14. Melloul E, Dondéro F, Vilgrain V, Raptis DA, Paugam BC, Belghiti J. Pulmonary embolism after elective liver resection: a prospective analysis of risk factors. J Hepatol 2012;57:1268-75.

15. Jeremitsky E, St. Germain N, Kao AH, Ong AW, Smith RS. Risk of pulmonary embolism in trauma patients: not all created equal. J Surg. 2013;154:810-5.

16. Stroh C, Michel N, Luderer D, Wolff S, Lange V, Köckerling F, et al, Risk of thrombosis and thromboembolic prophylaxis in obesity surgery: data analysis from the German Bariatric Surgery Registry. Obes Surg. 2016;26:2562-71.

17. Kokudo T, Uldry E, Demartines N, Halkic N. Pulmonary embolism, specific risk factor after pancreas resection? Pancreas. 2014;43:891-4.

18. Wang L, Pryor AD, Altieri MS, Romeiser JL, Talamini MA, Shroyer L, et al. Perioperative rates of deep vein thrombosis and pulmonary embolism in normal weight vs obese and morbidly obese surgical patients in the era post venous thromboembolism prophylaxis guidelines. Am J Surg. 